\title{
INFLUENCIA DEL MÉTODO DE ENSEÑANZA LA GLOBALIDAD EDUCATIVA EN EL RENDIMIENTO ACADÉMICO DE LOS ALUMNOS, PARA LA ASIGNATURA DE BIOLOGÍA, EN EL COLEGIO NACIONAL MIXTO FELIPE SANTIAGO ESTENÓS
}

\section{INFLUENCE OF THE METHOD OF EDUCATION THE GLOBALIDAD EDUCATIVA IN THE ACADEMIC YIELD OF THE PUPILS, FOR THE SUBJECT OF BIOLOGY, IN THE NATIONAL MIXED COLLEGE FELIPE SANTIAGO ESTENÓS}

\author{
Lic. Mario Posso Rojas
}

\section{RESUMEN}

El Método de Enseñanza la Globalidad Educativa (M.E.G.E), -caracterizado por el establecimiento de nexos entre las diferentes disciplinas por parte del alumnado en un determinado tema, perteneciente a una unidad didáctica de la asignatura de Biología- es una alternativa para mejorar el rendimiento académico. Empleando la distribución t $\mathbf{t}$ para muestras de varianza poblacional desconocida se obtuvo que el valor de $\mathbf{t}$ teórico, para un margen de error del $5 \%$ y 79 grados de libertad, es $\mathbf{t} \mathbf{0}=1,6641$ el que al ser contrastado con el valor de $\mathbf{t}$ calculado $\mathbf{t}=4,0481$, éste es mayor que el valor de $\mathbf{t}$ teórico, por lo tanto aceptamos la hipótesis alterna. Al culminar el trabajo de investigación se logró que los alumnos del $4 \mathrm{to}^{\circ} \mathrm{C}$ de la asignatura de Biología, además de obtener un mayor rendimiento académico, han potenciado sus capacidades intelectuales, motrices, artísticas y éticas.

Palabras Claves: Educación, M.E.G.E, rendimiento académico, prueba de hipótesis, potencia de capacidades y conclusiones.

\begin{abstract}
The Method of Education Globalidad Educativa (M.E.G.E), - characterized by the establishment of nexuses between the different disciplines on the part of the pupils in a certain subject, pertaining to a didactic unit of the subjet of Biology it is an alternative to improve the academic yield. Using t distribution, for samples of variance population not known obtained that the theoretical value of $t$, for a margin of error of $5 \%$ and 79 degrees of freedom, is t0 $=1,6641$ the one that to the being resisted with the calculated value of $t=4,0481$, this one is greater than the value of theoretical $t$, therefore we accepted the alternating hypothesis. When culminating the work of investigation was obtained that the students of 4 to ${ }^{\circ} \mathrm{C}$ of the subjet of Biology, besides to obtain a greater academic yield, have promoted their intellectual capacities, motor, artistic and ethical.
\end{abstract}

Key words: Education, M.E.G.E, test of hypothesis, academic yield, power of capacities and conclusions.

\section{INTRODUCCIÓN}

El bajo rendimiento académico es uno de los problemas, que en los últimos tiempos ha caracterizado a nuestro sistema educativo en las asignaturas de Lenguaje, Matemática y Ciencias Naturales, que nos han ubicado en el penúltimo lugar a nivel de Latinoamérica, y en el país con el mayor porcentaje de alumnos clasificados en el nivel inferior o por debajo.

Estos bajos resultados obtenidos se atribuyen al hecho que existe aún una mayoría de colegios en el Perú que siguen aplicando la metodología tradicional, que es rutinaria, vertical y no interrelaciona los temas.

La tarea del maestro, es ayudar a la educación del individuo, con el fin de perfeccionar su sociedad mediante la aplicación de métodos, técnicas e instrumentos que pertenecen a alguna teoría de aprendizaje; pero su verdadera misión no es sólo aplicar estos métodos, sino también formular su propio método de enseñanza.

En esta perspectiva presento el Método de Enseñanza la Globalidad Educativa, cuya sigla es M.E.G.E.
El M.E.G.E se carac-teriza por el estableci-miento de nexos entre las diferentes disciplinas, por parte del alumno, en un determinado

tema que pertenece a una unidad didáctica -ej. genética con física, química, geografía, historia, arte, lenguaje y otras- de una asignatura (Biología).

Las técnicas de enseñanza utilizadas están integrados; estas son: la Intradisciplina es la interrelación en la misma disciplina; la Interdisciplina es la interrelación o relación que se da entre una disciplina y otras afines; y la Multidisciplina que es la relación y a veces la interrelación que se da entre disciplinas o áreas que no son afines. A estas técnicas le acompañan los instruyentos, como el medio audiovisual, el mapa conceptual y el mental.

El M.E.G.E, parte del principio epistemológico de que hay similitudes entre todas las ramas del conocimiento que nos permite construir puentes entre las disciplinas, que puede ser estudiado por un experto multidisciplinario, pero sin olvidar su especialización en una determinada

\footnotetext{
a Licenciado en Educación, en la especialidad de Biología y Análisis Clínicos y Biológicos, Universidad Nacional de Educación (U.N.E). Creador del M.E.G.E. Maestría en Mejoramiento Genético de las Plantas, Universidad Nacional Agraria La Molina (UNALM). Escribe en http://educandos.es.t//Escribe-Mario-Posso.htm, http://marposroj1976.blogspot.com/ y consultor en Expertia.com.
} 
Los fundamentos para aplicar este método didáctico no sólo es filosófico, sino también biológicos, neuropsicológicos, psicológicos y didácticos, es por todas estas razones que estos fundamentos en conjunto nos dicen que se debe interrelacionar los temas en una asignatura, sin olvidar la especialización y teniendo en cuenta las formas y tipos de aprendizaje e inteligencia de los alumnos.

El objetivo de la investigación, es determinar la influencia del método de enseñanza la globalidad educativa en el rendimiento académico de los alumnos y alumnas de la asignatura de Biología, del cuarto grado "C", en el Colegio Nacional Mixto Felipe Santiago Estenós.

\section{MATERIAL Y MÉTODO}

Esta investigación es cuasiexperimental, y tiene un diseño de series temporales con un grupo, en la cual se utilizaron 2 métodos, el comparativo (examinar relaciones, semejanzas y diferencias de las notas obtenidas en las pruebas de entrada (E) con las de salida (S) y, a su vez, el promedio obtenido por el investigador con el profesor titular), y el analítico-explicativo (observación y comparación se establece las relaciones causales de los datos estadísticos).Y también 3 técnicas, el de observación (fichas de observación), la recolección de datos (pruebas de salida y entrada) y su tratamiento estadístico (media, varianza, desviación estándar, coeficiente de variación (C. V) porcen-tual, diferencia de medias, media y varianza conjunta, y distribución t) fueron utilizadas para demostrar la hipótesis.

En la metodología del trabajo, se consolidó siete temas, al inicio de cada una de ellas se evaluaron los conoci-mientos previos, denomi-nados pruebas de entrada y al finalizar cada tema se evaluaron los nuevos conocimientos adquiridos mediante el MEGE, denominándose prueba de salida.

Para ello se tuvo en cuenta una muestra de tipo no probalística, del Colegio Nacional Mixto Felipe Santiago Estenós, en la cual se eligió al $20 \%$ de la población estudiantil, eligiéndose el cuarto grado "C", del turno mañana, con 40 alumnos.

Las notas obtenidas fueron tratadas así:

$1^{\circ}$ En forma indepen-diente, las tres evaluaciones correspondientes al tercer bimestre y luego las cuatro evaluaciones correspondientes al cuarto bimestre, donde para cada evaluación se consideró la prueba de entrada con la prueba de salida.

$2^{\circ}$ Se consolidó las pruebas por bimestre.

$3^{\circ}$ Las notas de las pruebas de salida del tercer y cuarto bimestre, se trataron en forma comparativa con las notas del primer y segundo bimestre del profesor.

$4^{\circ}$ Para la explicación, se utilizaron la media, varianza, coeficiente de variación y la diferencia de medias.

Para la validación de la hipótesis se utilizó la diferencia de medias, empleando la distribu-ción $\mathbf{t}$ para muestras de varianza poblacional desconocida.

\section{RESULTADOS}

1) En el cuadro $N^{\circ} 1$ se muestran los resulta-dos de las evalua-ciones del tercer bimestre, en el que se aprecia que se obtuvo un mayor rendimiento en el tercer tema, con una media de 13,18, una diferencia de medias de $-3,90$. Así como disminuye el C. V $(28,83 \%)$ que representa una mayor homogeneidad en las notas obtenidas en las pruebas de salida del tercer tema.

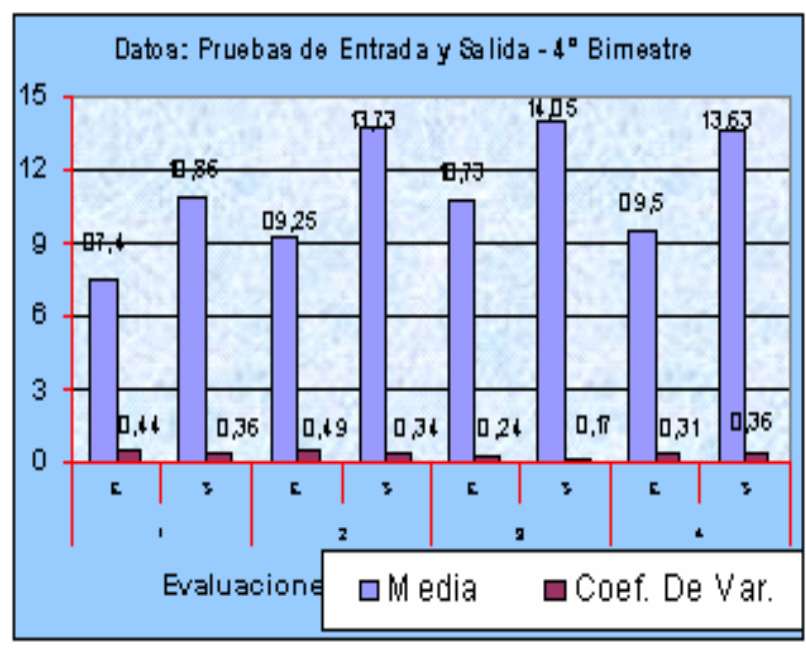

2) En el cuadro $\mathrm{N}^{\circ} 2$ se muestran los resulta-dos de las evaluacio-nes del cuarto bimes-tre, en el que se aprecia el rendimiento de los estudiantes es más homogéneo en la prueba de salida del tercer tema, con un promedio de 14,05 y una diferencia de medias de $-5,85$. Así como se incrementa la homogeneidad de las notas, $17,5 \% \mathrm{C}$. V.

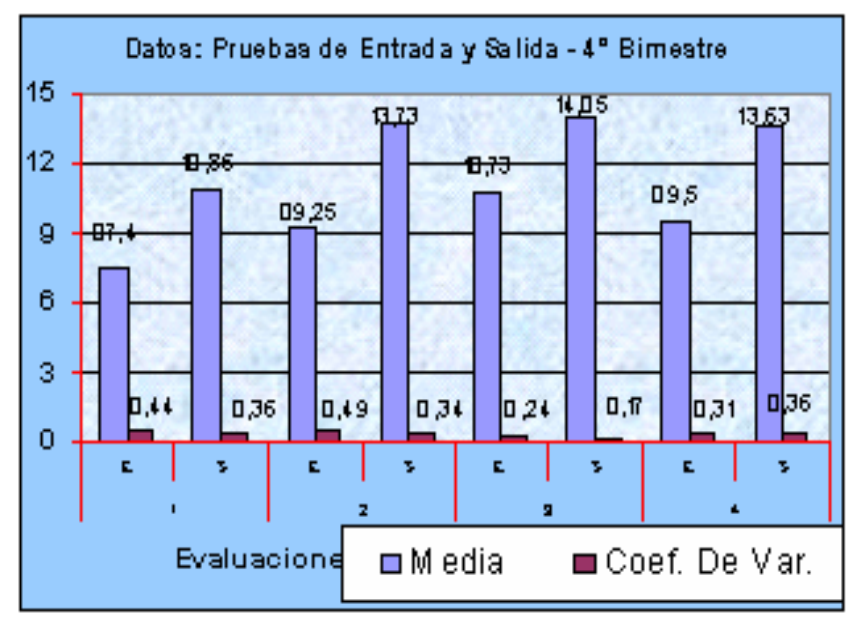

3) En el cuadro $\mathrm{N}^{\circ} 3$, se muestran los estadí-grafos correspon-dientes al tercer y cuarto bimestre, tanto de la prueba de entrada como de salida aplicadas por el investigador, en el que se observa que el rendimiento promedio de la prueba de salida del cuarto bimestre es mayor que la del tercero. También se obtuvo una mejor homogeneidad en el rendimiento académico $(19,84 \%$ C. V). 


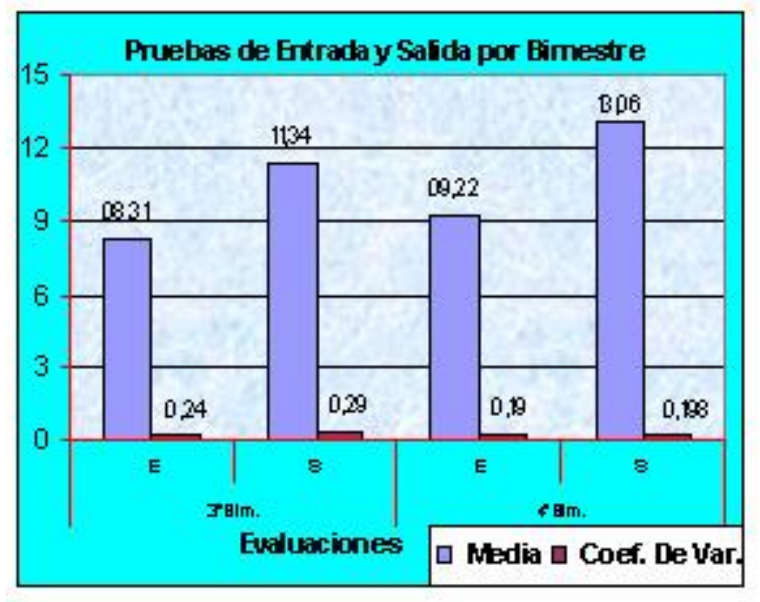

4) El cuadro $\mathrm{N}^{\circ} 4$, presenta los estadí-grafos de las pruebas de salida, en forma comparativa por bimestres, tanto los obtenidos por el profesor titular (primero y segundo bimestre) como por el investiga-dor (tercero y cuarto bimestre). En dicho cuadro observamos que de los dos bimestres conducidos por el profesor titular, es el segundo bimestre en que los alumnos tuvieron mayor rendimiento académico que la del primero $(12,48>8,00)$ y también fue más homogéneo $(19,30 \%$ de $C$. V frente a $39,12 \%$ de C. V.)

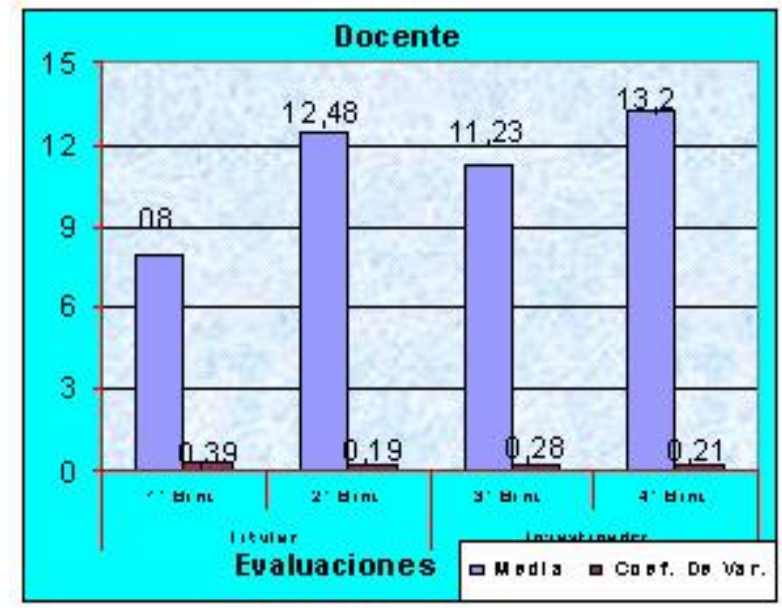

De los dos bimestres conducidos por el investigador se puede observar que en el cuarto bimestre los alumnos alcanzaron un mayor rendimiento académico que en el tercer bimestre $(13,2>11,23)$ y también el rendimiento fue más homogéneo en este último bimestre $(21,1 \%$ de C. V.).

Si comparamos los resultados obtenidos por el profesor titular y el investigador, podemos observar que con la aplicación del método de enseñanza la globalidad educativa que el investigador desarrolló, los alumnos obtuvieron un mayor rendimiento promedio $(13,2)$ en el cuarto bimestre. Como también se observa que de un promedio de 12,48 que obtienen en el segundo bimestre bajan a 11,23 en el tercero.

Este hecho pudo deberse al cambio del método de enseñanza tradicional a la globalidad educativa. Si bien es cierto que no se obtiene un promedio bastante significativo, puede decirse que en el cuarto bimestre el rendimiento promedio mejora, implicando esto la novedad del método de enseñanza la globalidad educativa.
5) En el cuadro $N^{\circ} 5$, se muestra los estadí-grafos de las notas de las pruebas de salida, en forma global, obtenidas por el profesor titular frente a las obtenidas por el investigador. Del que podemos afirmar que el rendimiento acadé-mico promedio de los alumnos del cuarto grado $\mathrm{C}$ de educación secundaria, del Colegio Nacional Mixto Felipe Santiago Estenós, mejora con la aplicación del método de enseñanza la globalidad educativa $(12,21>10,24)$ en comparación al rendimiento promedio obtenido por el profesor titular con la aplicación del método de enseñanza tradicio-nal. Obteniéndose una diferencia de medias de -3,73.

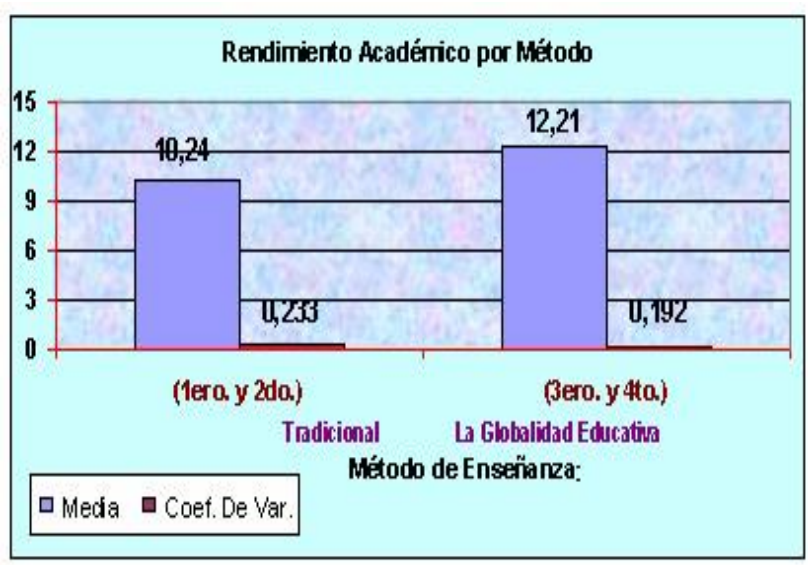

6) Del tratamiento descriptivo del resultado de las notas de las pruebas hemos podido observar que el método de enseñanza la globalidad educativa mejora el rendimiento académico de los alumnos del Colegio Nacional Mixto Felipe Santiago Estenós en la asignatura de Biología.

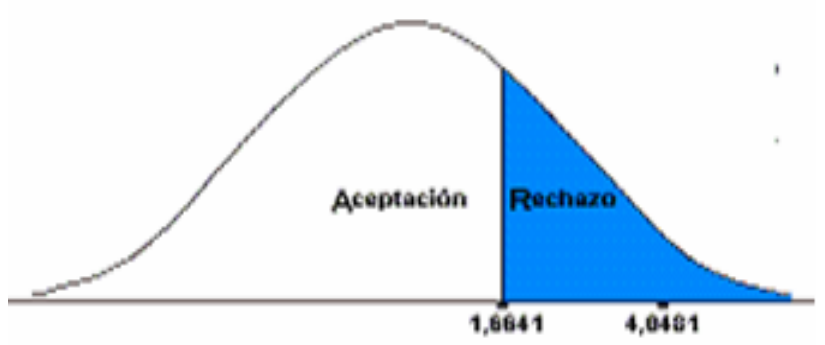

Gráfico H" 6: "Contraste de las Himótesis"

Para verificar la hipótesis alterna $\mathbf{H}_{\mathbf{1}}$ que dice: Con la aplicación del método de enseñanza la globalidad educativa se logra un mayor rendi-miento académico en los alumnos y alumnas de la asignatura de Biología, del cuarto grado "C", del Colegio Nacional Mixto Felipe Santiago Estenós $\left(\mu_{1}>\mu_{2}\right)$.

Se empleó la distribu-ción t, para muestras de varianza poblacional des-conocida se obtuvo que el valor de $\mathbf{t}$ teórico, para un margen de error del $5 \%(\alpha=0,05)$ y 79 grados de libertad, es $\mathbf{t}_{0}=1,6641$ el que al ser contrastado con el valor de $\mathbf{t}$ calculado $\mathbf{t}=4,0481$, éste es mayor que el valor de $t$ teórico; tal como se muestra en el gráfico $N^{\circ} 6$. Como $\quad t=4,0481$ cae en la zona de rechazo, rechazamos la hipótesis nula $\mathrm{H}_{0}$ y aceptamos como válida la hipótesis alterna $H_{1}$. Conclusión que fue corrobora-do por SPSS. Esto nos permitió rechazar $\mathbf{H}_{0}$ y considerar como válida $\mathbf{H}_{\mathbf{1}}$. 
Al culminar el trabajo de investigación se logró que los alumnos del $4 \mathrm{to}^{\circ} \mathrm{C}$ de la asignatura de Biología, durante los dos últimos bimestres del año 2001, además de obtener un mayor rendimiento académico, han potencia-lizado sus capacidades intelectuales, motrices, artísticas y éticas.

\section{DISCUSIÓN}

El M.E.G.E facilitó, a los alumnos y alumnas, conocer en forma integral las diversas estructuras orgánicas y los procesos hereditarios, del ser humano y de otros organismos.

El M.E.G.E completen-tado con la indagación, la evaluación, la aplicación de lo aprendido y la retroalimentación; hace que los alumnos y alumnas retengan con mayor éxito los conoci-mientos en forma activa.

Con las técnicas de enseñanza de este método: la intradisciplina, la interdisciplina, y la multidisciplina en conjun-to, se logró que el alumno y alumna conozca las estructuras orgánicas de su cuerpo y de los otros seres vivos con mayor dinamismo y claridad.

Los promedios obteni-dos en el tercer bimestre con las pruebas de salida $(11,34)$, con la aplicación del método de enseñanza la globalidad educativa, son mayores que con las pruebas de entrada $(08,31)$; y también en el cuarto bimestre los promedios obtenidos en las pruebas de salida $(13,06)$ son mayores que los de las pruebas de entrada $(09,22)$.
Con la aplicación del método de enseñanza la globalidad educativa se logró un mayor rendimiento académico (t es 4,0481) en los alumnos y alumnas, de cuarto "C", de la asignatura de Biología del Colegio Nacional Mixto Felipe Santiago Estenós.

Se recomienda a los maestros de Biología, y de otras especialidades, aplicar el M.E.G.E en sus asignaturas para lograr la participación de todos los alumnos y las alumnas debido a la correlación entre los conocimientos teóricos y prácticos.Para optimizar mejores resultados con la aplicación del M.E.G.E deberá estructurarse una programación en todo el año, con ayuda del Currículo Integral adoptado a la educación secundaria.

\section{AGRADECIMIENTOS}

Un reconocimiento muy especial a mi asesora, Biolg. Flor de María Wong Baquero; a la directora del Colegio Nacional Mixto Felipe Santiago Estenós, la Lic. María Oré Guzmán; al Lic. Noé Rafael Mendoza profesor de la asignatura de Biología; a los alumnos y alumnas del 4to. C; a los docentes Ms. Ed. Guillermo Arévalo del Aguila (q.e.p.d.), a la Mg. Dora Mesias Borja; al Dr. Walter Peñaloza Ramella. (q.e.p.d.), a la Dra. Ligia Gutiérrez Deza; al Lic. Genaro Quispe Pineda, Lic. Ramiro Butarra; a mi padre Mario Rufino Posso Ñañez, a mi tíos Nicolás y Petronila, a mis hermanas Margarita y Karina. $Y$ todas las demás personas que siempre me ayudan a ser mejor profesional y persona cada día.

\section{REFERENCIAS BIBLIOGRAFICAS}

1. Ary, D.; Jacobs, C. L; Razavieh, A. Introducción a la Investigación. Ed. Interamericana. México - México. Segunda edición. 1985.410 pp.

2. Eccles, John C. El Cerebro. Morfología y dinámica. Ed. Interamericana, México - México. 1975. 236 pp.

3. Ferrière, Aldo. La Escuela Activa. Ed. Francisco Beltrán, Madrid - España. 1927. 344 pp.

4. Posso Rojas, Mario. Influencia del método de enseñanza la globalidad educativa en el rendimiento académico de los alumnos, para la asignatura de biología, en el Colegio Nacional Mixto Felipe Santiago Estenós. Tesis 2004. U.N.E, 150 pp.

5. Torrejon Reyna, Luis. Técnica de la Globalización. Ed. Escuela Normal Superior, Chosica - Perú. Segunda edición. 1966. 211 pp.

6. Zabala Vidiella, Antoni. Enfoque Globalizador y Pensamiento Complejo: Una respuesta para la comprensión e intervención en la realidad. Ed. GRAO. Barcelona - España. 1999. 201 pp.

Email: marposroj@hotmail.com 